Reviews

\title{
Assisted reproductive technologies - is it gaining the upper hand over natural physiology?
}

\author{
R M C B Hunukumbure ${ }^{1}$ \\ Sri Lanka Journal of Obstetrics and Gynaecology 2011; 33: 116-120
}

\section{Introduction}

For most people the desire to have children is fulfilled naturally. However, about $10-15 \%$ of couples experience difficulties in achieving pregnancy as planned $^{1}$. Infertility is a widespread condition and is regarded as a psychologically stressful by most individuals and can lead to depression, social isolation and poor quality of life $^{2}$.

In vitro fertilization (IVF) is where mature oocytes are surgically removed from the ovary and then fertilized with sperm in the laboratory. Today $2-3 \%$ of all newborns in many countries are conceived with the help of in vitro fertilization.

The immense complexity surrounding the fertilization process means that several key issues need to be addressed before successful IVF becomes a reality. These include the control of the oocyte maturation process, retrieval of the oocytes at a developmental stage suitable for in vitro fertilization, activation of the sperms in vitro, creation of conditions that would allow fertilization as well as the early development of the embryos in vitro, and the transfer of the embryo/ embryos back in to the mother's uterus for further development.

Ovulation is the first step to be identified for the conquest of infertility, as it is a prerequisite for pregnancy in the female. It is the result of a delicate, well synchronized balance between the central nervous system, hypothalamus, pituitary and the ovary $^{3}$. Being the central episode of the ovarian cycle, it involves a complex and a multifaceted process that culminates in the rupture of the dominant follicle leading to the release of a fertilizable oocyte ${ }^{4}$.

The hypothalamus occupies a key position in the control of the ovarian function. Its control is exerted

\footnotetext{
${ }^{1}$ Senior Registrar in Subfertility, Professorial Unit in Obstetrics and Gynaecology, De Soysa Hospital for Women, Colombo 8, Sri Lanka.

Correspondence: Chaminda Hunukumbure

E-mail: rmcbhunu@yahoo.com
}

by secretion of the deca-peptide gonadotrophin releasing hormone $(\mathrm{GnRH})$, which in turn stimulate the secretion of both follicular stimulating hormone (FSH) and leutinizing hormone (LH) secretion by the gonadotrophs of the anterior pituitary.

In the natural ovarian cycle, the initiation of the follicular growth is a continuous process that is believed not to depend on hormonal stimulation. Each day, about fifty follicles begin to grow. The majority undergo rapid atresia. The total growth phase extends over a period of ninety days. Towards the end of the preceding cycle, luteolysis takes place under the influence of prostaglandins (PGF $2 \alpha$ ) and probably endothelins (ET-1), and the resulting fall in the circulating oestrogens, progesterone and inhibin B leads to a rise in the gonadotrophin level. At the beginning of each new cycle, a crop of follicles respond to the rising FSH level by entering the pre-antral phase $^{5}$. Each follicle, which contains an immature ovum, contains granulosa cells and theca cells in its periphery. The granulosa cells start to secrete $17 \beta$ oestradiol (E2) by way of aromatizing the androgen testosterone produced by the theca cells. E2 and FSH together cause proliferation of the granulose cells and increase the number of FSH receptors on the granulsa cell membrane ${ }^{5}$. Based on its ability to convert androgens into oestrogens, a dominant follicle is selected and the remaining follicles of the cohort that began developing at the commencement of the cycle undergo atresia.

$\mathrm{FSH}$ is responsible for the early maturation of the ovarian follicles, and FSH and LH together are responsible for their final maturation. In addition, numerous intra ovarian peptides including insulinlike growth factor-1, inhibins, activins, follistatins and growth factors such as vascular endothelial growth factor, epidermal growth factor, and transforming growth factor $\alpha$, function in paracrine and autocrine fashion to modulate the effects of the gonadotrophins. A burst of LH secretion is responsible for ovulation and the initial formation of the corpus luteum. 


\section{Down regulation}

Although ovulation induction agents were available since the 1960s, the monumental work of Edwards and Steptoe that resulted in the birth of Louise Joy Brown in 1978 was carried out on the natural cycle, since they were convinced that the hormone stimulation protocols available at that time disturbed the implantation of the embryo in the uterus leading to spontaneous miscarriage. Furthermore the first successful pregnancy that they achieved in 1976 had to be terminated because it was a tubal ectopic pregnancy. Although non simulated cycles are known to be safer and reduce the costs, and has been claimed to reduce aneuploidy rates by its proponents, the pregnancy rates are merely around $10 \%$. The answer to this was the rejuvenation of the ovarian stimulation protocols which was pioneered by the Monash Group. However, the common problem with these protocols were that the presence of endogenous gonadotrophins led to premature leutinization in $30-40 \%$ of the cycles, and in many others, ovulation occurred at an inconvenient time of the day, resulting in cycle cancellation ${ }^{5}$. The major step in circumventing these unwanted phenomena was the introduction of GnRH analogues.

The use of gonadotrophin releasing hormone (GnRH) agonists aids to improve the controlled ovarian stimulation protocols by way of permitting more synchronous development of follicles. GnRH agonists significantly reduce the likelihood of premature LH surge. They also alleviate the effects of progesterone on the developing follicles. GnRH agonists bind to pituitary GnRH receptors and stimulate LH and FSH synthesis and release. However, the agonists have a much longer biologic half life ( 3 to 8 hours) compared to endogenous GnRH (3.5 min). The prolonged exposure thus created leads to loss of pituitary receptors and down regulation of the GnRH activity, drastically reducing serum FSH and LH levels for as long as the drug is given. The menopausal state thus created provides the ideal platform for controlled ovarian stimulation. These agents can be administered intranasally, or subcutaneously daily or as subcuticular depot preparations.

More recently GnRH antagonists have come into the forefront of IVF protocols. These agents act by competitive inhibition of the $\mathrm{GnRH}$ receptors and produce an immediate suppression of pituitary gonadotrophin release without inducing an initial stimulatory effect; hence they can be started after the ovarian stimulation with gonadotropins has begun. GnRH antagonists thus allow shorter IVF protocols and renders greater flexibility to the stimulation regime. They act primarily to prevent a premature LH surge. They are also devoid of the distressing menopausal side effects produced by their agonist counterparts.

A study conducted on 3000 women undergoing IVF-ET with or without different regimens of GnRH agonist Buserelin revealed that the cumulative live birth rate was significantly higher among patients who were subjected to down regulation protocols ${ }^{6}$. This phenomenon was related to the improvement in the synchronization of follicular development leading to a larger cohort of mature follicles at the time of retrieval, while others have postulated that it is due to the longer stimulation with gonadotrophins observed in $\mathrm{GnRH}$ agonist cycles.

The use of monophasic preparations of oral contraceptive pills before the start of IVF cycles renders greater flexibility in the planning of cycle start days. The patients receiving oral contraceptive pills are less likely to have large ovarian or corpora leuteal cysts at the beginning of the IVF cycle which could delay the cycle initiation. It also prevents spontaneous ovulation ${ }^{7}$.

Over the last 25 years the stimulation protocols have been refined and now include.

\section{Agonist cycles - long protocol}

The start of the GnRH agonist administration is either on day 2 of the menstrual cycle, or more commonly on day 21 of the previous cycle. In their research Urbanscek and Wtthaus found that the clinical pregnancy rate and the live birth rate were better when a GnRH agonist was started in the mid luteal phase rather than in the early follicular phase. The temporary menopause this creates allows ovaries to be stimulated using FSH or HMG injections.

\section{Antagonist cycles - short protocol}

The GnRH antagonists are administered either on a fixed day of the FSH stimulation (usually on the 5 th day) or when the lead follicle has attained a certain size by ultrasound monitoring, and continued alongside gonadotrophin stimulation until an adequate response is obtained ${ }^{8}$.

Short protocols with GnRH agonists has lost popularity due to poor success rates.

\section{Controlled ovarian stimulation}

The process of creating a cohort of co-dominant follicles suitable for oocyte retrieval on days 11-15 using medications is called "controlled ovarian stimulation". This stimulation is achieved through 
the administration of medications such as clomiphene citrate, human menopausal gonadotrophins (HMG), or follitropin (FSH). Some formulations contain FSH and LH, while others contain FSH only ${ }^{7}$. Newer more purified products permit subcutaneous, rather than intramuscular administration, and recombinant techniques allow for easier control of purity and consistency of the preparations ${ }^{9}$. Daily dosing is the usual practice. This results in patterns of gonadotrophin levels markedly dissimilar from those seen in the course of normal ovarian physiology (lack of pulsatility, achievement of a single maximum over 24 hours approximately 12 hours after injection, and gradual accumulation over the days of follicle recruitment). Despite this, daily injection has not been shown to be inferior to more frequent or pulsatile administration 9 . Pharmacokinetics after intra muscular or subcutaneous administration of recombinant preparations is similar, even in obese women.

Achievement of ovulation is not the principal challenge in ovulation induction with gonadotrophins; rather it is the achievement of a response that is safe with regard to the risk of ovarian hyperstimulatin syndrome (OHSS). Monitoring for a follicular response predicting fertile ovulation, but not excessive response is essential. This relies on a combination of biophysical and hormonal data? Serial ultrasound scans, usually through the transvaginal route are used for the determination of the number and the size of the developing follicles. The assessment of the ovarian volume and the ancillary findings such as presence/absence of free fluid in the pelvis contribute to rule out ovarian hyperstimulation. Follicular stimulating hormone (FSH) stimulates the secretion of oestradiol by the granulosa cells in the developing follicles by increasing their aromatase (CYP 19) activity, and the serum oestradiol level presents an excellent bioassay for ovarian stimulation with exogenous gonadotrophins. Methods for assaying the steroidal hormones in the blood include enzyme-linked immunosorbent assay and radioimmunoassay. Oestogen assays have been shown to allow for reliable comparisons. However, a decreasing number of units use serial oestradiol levels since it is cumbersome to the patient and its place has been taken over by serial transvaginal ultrasound scans. In fact NICE Guideline states that serum oestradiol monitoring provided no additional information compared to ovarian ultrasound. All the same serial oestradiol levels can be useful in some patient groups, particularly if an over or under-response is anticipated ${ }^{8}$.

\section{Ovulation}

The mid cycle gonadotrophin surge of LH and FSH from the pituitary is the initiator of ovulation.
The LH component of the gonadotrophin surge is the main trigger. Several studies have clearly shown that $\mathrm{LH}$ is the physiological trigger of ovulation by binding to the $\mathrm{LH}$ receptors. The $\mathrm{LH}$ receptors are present in high density on theca cells and the mural granulosa cells, but the density on the cumulus granulosa cells is substantially lower ${ }^{4}$. Though it may appear physiological to use exogenous LH to trigger ovulation, recombinant $\mathrm{LH}$ is not commercially available. This problem is successfully dealt by the use of the glycoprotein hormone human chorionic gonadotrophin (hCG) which, like other glycoprotein hormones is made-up of $\alpha$ and $\beta$ subunits. hCG- $\alpha$ is identical to the $\alpha$ subunits of LH and FSH and it is primarily lutinizing and luteotrophic with little FSH activity $^{10}$. Under natural circumstances ovulation occurs 36 hrs after the onset of the LH surge. Therefore the administration of hCG should be planned in such a way that oocyte retrieval could be performed prior to physiological ovulation takes place.

When the lead follicles reach a mean diameter of $18 \mathrm{~mm}$, and if measured risk for OHSS is permissive, ovulation is triggered with administration of 10000 international units of hCG intramuscularly (or 5000 IU in patients with a history of over response). This is timed in such a way that oocyte retrieval is carried out approximately 34 hrs later.

In 1969, Dr. Robert G. Edwards made an important breakthrough in the field of reproductive research when he discovered that under specific buffer conditions, activated human spermatozoa could promote fertilization of in-vitro matured oocytes. Although this provided crucial insights into research on human reproduction, the major drawback was the failure of the in-vitro matured oocytes to develop beyond two cell stage once they were fertilized. The way forwards seemed to be the use of oocytes that had completed maturation in vivo. His earlier work had shown that the meiotic maturation of the oocytes could be initiated and controlled by exogenous gonadotrophins and he knew from the in vitro studies that he had conducted on human oocytes, the timing of the meiotic maturation process in humans. The major obstacle remained was the method of oocyte retrieval. Dr. Edward's quest to find a solution for this problem lead to his collaboration with Dr. Patrick Steptoe, Obstetrician and Gynaecologist, who had pioneered the use of laparoscopy in England. The end result was the introduction of laparoscopic oocyte retrieval.

Today with advances in real time ultrasound technology, especially with the advent of smaller and better quality trans vaginal probes, oocyte retrieval is generally done under trans vaginal ultrasound 
guidance, unless the ovaries are not assessable through the trans vaginal route due to previous surgical ovarian transposition. Trans vaginal oocyte retrieval is typically performed using conscious sedation, which allows it to be carried out as an office procedure.

Depending on sperm quality, oocytes may be inseminated using routine fertilization where the oocyte is placed with sperms in a petry dish or through micromanipulation techniques, such as intracytoplasmic sperm injection (ICSI). Sperms are prepared by washing and centrifugation. For routine fertilization, 50000 to 100000 motile sperms are placed in a petry dish with an oocyte. A major discovery in assisted reproductive techniques is that sperm need not be added to the oocytes immediately after retrieval, and a delay of 4 to 6 hours improves fertilization rates. Fertilization can be detected 12 to 24 hours after insemination by the presence of two pronuclei found in the cytoplasm of the oocyte and the presence of two polar bodies in the perivitelline space. Twenty four hours after insemination, the pronuclear membranes dissolve allowing combination of the maternal and paternal chromatids (syngamy), which is followed by the first cleavage division ${ }^{7}$.

Since its addition into the armory of assisted reproductive techniques by Van Steirteghem and coworkers, intra-cytoplasmic sperm injection (ICSI) has revolutionized the practice of IVF. Until then severe male factor infertility was considered a challenge even for conventional IVF. With ICSI a single sperm is injected through the zona pellucida into the mature oocyte. The possibility of achieving pregnancy with only a single sperm is truly fascinating. Sperm used in ICSI are not only obtained from the ejaculate, but also in men with no sperm in ejaculate by using micro surgical epididymal sperm aspiration or testicular biopsy ${ }^{7}$.

With the advancement of the knowledge of the micro environment suitable for the embryo to develop in vitro, it became evident that the metabolic requirements of the developing embryo changes with the advancing gestational age. Therefore, single stage or two stage tissue culture media will be required depending of the timing of the embryo transfer along with stringent control of the incubator environment in relation to temperature, $\mathrm{pH}$, air quality and oxygen tension in order to facilitate optimal results.

An amazing discovery with IVF was that the embryos are capable of completing their development in vitro. Another ground breaking discovery was the biotechnology associated with extraction of a blastomere from the embryo without compromising its further development. The advent of DNA amplification techniques (polymerase chain reaction) has paved the way for pre-implantation genetic diagnosis. It provides an alternative to prenatal diagnosis for couples who run a substantial risk of producing offspring with many known genetic disorders.

\section{Embryo transfer}

In the natural cycle, fertilization occurs in the ampulla of the fallopian tube. As the developing embryo is taken towards the uterine cavity it undergoes further cell division, compaction with the formation of inner and outer cell masses, and by the time it reaches the uterine cavity becomes the blastocyst comprising the blastocele, embryoblast and the trophoblast. In IVF, embryos can be transferred to the uterus on day 2,3 or 5 after insemination, by which time they have developed into two, four or eight cell stage morula/blastocyst stage. Day 2 transfer may appear unphysiological since in the natural cycle the embryo would be still in the fallopian tube but it renders the advantages of the use of a single culture medium, the majority of the fertilized ova are available for transfer which also provides surplus embryos for cryopreservation. Day 5 transfer which involves the blastocyst stage appears more physiological, and provides the best surviving embryos for transfer but there may not be any embryos left for cryopreservation.

A variety of soft, plastic embryo transfer catheters are used for embryo transfer which are inserted through the cervix into the uterine cavity. Embryo transfer should be performed under trans abdominal ultra sound guidance as this allows more accurate placement of the embryos in the uterine cavity and has been shown to increase success rates significantly ${ }^{8}$.

\section{Luteal support}

Luteal function may be truncated in patients without sufficient endogenous LH, and this is typically corrected either with supplemental progesterone or a booster injection of hCG several days after the ovulatory dose of hCG is given. In addition leuteolysis is known to be associated with certain IVF regimens. Owing to this some form of luteal phase support is generally considered necessary. Luteal phase support (LPS) has been shown to improve success rate even with antagonist cycle where there is a controversy about its usefulness ${ }^{8}$. LPS comes in two forms. First the use of luteolytic agents such as hCG which stimulates patient's own ovaries to produce more progesterone. The second is the use of progestogens or progesterone. LPS is continued for a minimum of two weeks but some clinics routinely advocate it up to 12 weeks or even later. 


\section{Cryopreservation}

Controlled ovarian stimulation protocols results in the production of multiple oocytes, leading to multiple embryos. Transfer of multiple embryos to the uterus may increase the success rate, but it also increases the incidence of multiple pregnancies, which is associated with numerous complications for both the mother and for the fetuses. The number of embryos transferred will depend on the patient's age, the ovarian reserve and the prior history ${ }^{7}$. During the last decade, the trend has been towards transferring fewer embryos. For this to become a reality there should be reliable and effective preservation techniques for the supernumerary embryos which could be transferred in subsequent cycles. Hence the emergence of cryopreservation.

The first pregnancy using cryopreserved embryos from an earlier IVF cycle was reported in 1984. Cryopreservation involves a series of complex and dynamic physiochemical processes related to temperature and water transport between a cell and the surrounding medium. The basic goal of cryopreservation is to achieve intracellular vitrifaction while avoiding intracellular ice formation and membrane and organelle damage ${ }^{11}$. In general, cryopreservation techniques related to human embryos and oocytes fall into two categories; Controlled slow freezing and vitrifaction. Slow freezing attempts to maintain a delicate balance between cryoprotectants and the aqueous embryo compartment whereas the strategy for vitrifaction method is a rapid solidification of liquid without ice crystal formation ${ }^{11}$.

In contrast to the success of embryo freezing, which is considered routine, the cryopreservation of the oocytes has been less successful. Only a few births have been achieved since this technique was first introduced in 1986.

Cryopreservation of excess spermatozoa is also a possibility, following surgical retrieval of spermatozoa prior to IVF/ICSI in obstructive causes of male infertility. It obviates the need for further surgery in subsequent cycles.

\section{Conclusion}

In vitro fertilization and its related reproductive technologies have brought immense relief to the untold suffering of millions of infertile couples worldwide. In order to achieve this target we have made numerous in roads into natural physiology. However, there is a genuine concern whether in our quest for success we are challenging the delicate balance of nature by way of altering phenomena such as Natural Selection and this has created numerous ethical issues.

\section{References}

1. Embryology/IVF. http://www.nikoninstruments.com/ Applications/Biological/Clinical/Embryology-IVF (Accessed on 12 th June).

2. Johansson M, Adolfsson A, Berg M, Frances J, Hogström L, Janson PO, Sogn J, Hellström AL. Quality of life for couples 4-5.5 years after unsuccessful IVF treatment. Acta Obstet Gynecol Scand 2009; 88: 291-300. http:// on linelibrary.wiley.com / d oi / 10.1080 / 00016340802705956/pdf (Accessed on 3rd of June).

3. Begum MR, Quadir E, Begum A, Begum RA, Begum M. Role of aromatase inhibitor in ovulation induction in patients with poor response to clomiphene citrate. J Obstet Gynaecol 2006; 32(5): 502-6.

4. Brännström M, Lind $\mathrm{AK}$, Kähler PD. Ovulation: a molecular view. In: Douglas T, Carrell C, Matthew P (eds). Reproductive Endocrinology and Infertility. Longdon: Springer; 2010: 119-32.

5. Basics of ovarian stimulation. http://ivf-egypt.com/ about\%20ivf/basic.aspx\#top (Accessed on 12 th June).

6. Palermo G, Joris H, Derde MP, Camus M, Devroey P, Van Steirteghem AC. Sperm characterization and outcome of human assisted fertilization by subzonal insemination and intracytoplasmic sperm injection. Fertil Steril 1993; 59: 826-35.

7. Deborah L, Manzi-Smith. Assisted reproductive technologies. In: Alvero, Schlaff. Reproductive Endocrinology and Infertility. Philadelphia: Mosby Elseveir; 2007: 263-81.

8. Trew G. Assisted reproduction. In: Edmonds DK (ed). Dewhurst's Textbook of Obstetrics and Gynaecology, 7th edition. Massachusetts: Blackwell publishing; 2007: 461-77.

9. Mark Gibson . Ovulation Induction. In: Douglas T, Carrell C, Matthew P (eds). Reproductive Endocrinology and Infertility. Longdon: Springer; 2010: 525-36.

10. The gonads: development and function of the reproductive system. In: Barrett KE, Barmen SM, Boitano, Brooks HL. Ganongs's Review of Medical Physiology, 23rd edition. New York: Tata McGraw Hill Publishing; 2010: 391-428.

11. Behr B, Shu Y. Cryopreservation of human oocytes and embryos. In: Douglas T, Carrell C, Matthew P (eds). Reproductive Endocrinology and Infertility. Longdon: Springer; 2010: 689-98. 